\title{
Propriétés d'un circuit graphe minimum
}

\author{
Jean Pierre Nzali \\ Département d'informatique \\ Université de Yaoundé I \\ BP 812, Faculté des Sciences \\ CAMEROUN \\ jpnzali@uycdc.uninet.cm
}

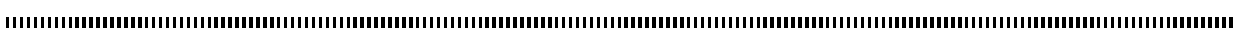

RÉSUMÉ. Un circuit graphe est un graphe planaire topologique dont les arcs sont orientés de telle sorte que chaque face finie soit un circuit. Il est minimum si le nombre d'arcs orientés dans les deux sens est minimum. Dans cet article nous étudions les propriétés d'un tel graphe. Nous montrons que chaque face finie peut être caractérisée par son sens d'orientation. Nous présentons aussi quelques résultats sur la disposition des arcs orientés dans les deux sens sur un circuit graphe minimum

ABSTRACT. A graph circuit is a planar topologic graph in which edges are oriented such that any finite face is a circuit. Such graph is said to be minimum if the number of edges oriented in two direction is minimum. In this article we study such graphs properties. We prove that each finite face can be characterized by the his orientation direction. We also present sum results on the disposition of edges oriented in two directions in a minimum graph circuit.

MOTS-CLÉS : graphe planaire topologique, circuit graphe, carte, degré de retournement, problème du postier chinois.

KEYWORDS: planar topologic graph, graph circuit, map, reversal degree, chinese postman problem.

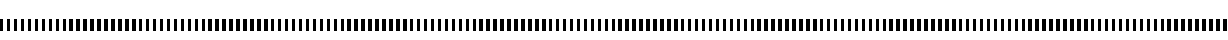

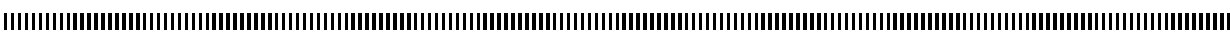

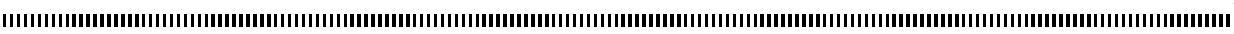




\section{Introduction}

Dans les pays en développement, bien que l'apport des Systèmes d'Informations Géographiques (SIG) soit indéniable comme partout ailleurs [6], [7], [16], surtout dans ses aspects d'aide à la décision et maîtrise de l'environnement, le manque de matériel adapté à la saisie des données spatiales constitue un véritable frein à l'introduction de cette nouvelle branche de l'informatique. Les notions de degré de retournement et de circuit graphe minimum que nous étudions ici sont issues de l'acquisition, pour un logiciel de SIG, de cartes par une méthode n'utilisant pas les outils traditionnels comme le scanner ou la table à digitaliser. Cette méthode appelée Digitalisation au Millimètre (DIMI) a été expérimentée sur la carte du Cameroun ([5]) et a donné des résultats satisfaisants, particulièrement dans le cadre des applications en cartographie thématique. La méthode et les résultats obtenus ont été présentés dans [10], [11]. La connaissance du degré de retournement et des propriétés d'un circuit graphe minimum facilite l'acquisition par la DIMI de la carte associée. Les propriétés d'un circuit graphe minimum sont surtout intéressantes dans les SIG fonctionnant selon le modèle vecteur topologique [6] où chaque arc est rangé une seule fois dans la base, mais doit être lu dans un sens ou dans un autre selon la face dans laquelle il s'incorpore. En effet dans une carte chaque face est constituée d'un certain nombre d'arêtes. La face est obtenue en parcourant l'ensemble de ces arêtes dans un certain sens qui ne sera pas forcément le même pour les faces voisines.

Le degré de retournement est une caractéristique des graphes planaires topologiques ou cartes. Nous l'avons étudié et nous avons proposé des approches de son calcul dans [11], [13] et [14]. Dans ces précédents travaux, nous avons montré que le degré de retournement est une fonction entre autre du nombre de sommets intérieurs de degré impair (sidi), de la disposition de ces sidis les uns par rapport aux autres ainsi que de leur profondeur (nombre d'arc minimum qui relie un sidi à un sommet extérieur). Les algorithmes présentés jusque là reposent sur les notions de sidi, de la profondeur de chaque sidi et de la disposition des sidis les uns par rapport aux autres. Ces algorithmes présentent une explosion combinatoire ([8], [13]) avec un nombre de cas à étudier supérieur à $\mathrm{e}^{\mathrm{n}}$ (pour $\mathrm{n}>12$ ), $\mathrm{n}$ désignant le nombre de sidi. Pour résoudre ce genre de problème on a souvent recours à des heuristiques. Comme nous partons d'une carte non orientée et que le résultat est une carte orientée de telle sorte que chaque face soit un circuit, (d'où le nom de circuit graphe minimum), bien connaître les propriétés d'un circuit graphe minimum devrait suggérer des heuristiques et des approches de solution.

Cet article présente quelques propriétés importantes des cartes orientées de telle sorte que chaque face soit un circuit. Dans la suite nous commençons par quelques rappels et définitions de la théorie des graphes avant d'aborder les propriétés des circuits graphe minimums. 


\section{Définitions.}

\subsection{Graphe planaire et carte}

Un graphe $\mathrm{G}$ ([1], [3], [4]) est un couple (X,E) formé de deux ensembles disjoints $X=\left\{x_{1}, x_{2}, \ldots, x_{n}\right\}(n>0)$ et $E=\left\{e_{1}, e_{2}, \ldots, e_{m}\right\}(m>0)$ tel que pour tout $i, e_{i}$ est une paire d'éléments de $\mathrm{X}$. X est appelé ensemble de sommets et $\mathrm{E}$, ensemble d'arêtes. Une chaîne d'un graphe $G$ est une suite alternée de sommets et d'arêtes $G=x_{0} e_{1} x_{1} e_{2} \ldots x_{k}$ ${ }_{1} e_{k} x_{k}$ telle que pour tout $1 \leq \mathrm{i} \leq k$, les extrémités de $e_{i}$ sont $x_{i-1}$ et $x_{i}$. Un graphe $G$ est connexe si et seulement s'il existe une chaîne entre deux sommets quelconques de G.

Un graphe $\mathrm{G}$ est un graphe planaire s'il est possible de le représenter sur un plan de telle sorte que les sommets soient des points distincts, les arêtes des courbes simples et que deux arêtes ne se rencontrent pas en dehors de leurs extrémités. Sa représentation sur un plan est appelée graphe planaire topologique ou carte. Dans la suite de l'article nous nous intéresserons uniquement aux graphes planaires topologiques connexes sans isthme et sans point d'articulation.

Un isthme est une arête dont la suppression augmente le nombre de composantes connexes. De même un point d'articulation d'un graphe $\mathrm{G}$ est un sommet de G qui, s'il est enlevé, augmente le nombre de composante connexe de G. Quand nous parlerons de carte $K$ il s'agira toujours d'un graphe planaire topologique connexe, sans isthme et sans point d'articulation.

Une face d'une carte $\mathrm{K}$ est une région du plan limitée par des arêtes et telle que deux points arbitraires dans cette région peuvent toujours être reliés par un trait continu ne rencontrant ni sommets ni arêtes. Deux faces d'une carte sont voisines si elles ont une arête commune qui les délimite. Il y a toujours une face non entièrement délimitée appelée face infinie. Les autres faces sont des faces finies. Une face finie sera dite extérieure si elle est voisine de la face infinie. Dans le cas contraire elle sera dite intérieure.

Une arête est incidente à un sommet si ce sommet est une des extrémités de cette arête. Le degré d'un sommet est le nombre d'arêtes incidentes à ce sommet. Nous dirons qu'un sommet $\mathrm{S}$ de $\mathrm{K}$ appartient à une face $\mathrm{F}$ si et seulement si deux arêtes appartenant à la frontière de $\mathrm{F}$ sont incidentes à ce sommet. Comme la carte $\mathrm{K}$ ne possède pas d'isthme, chaque arête délimite deux faces distinctes finies ou non. Comme K n'a pas non plus de point d'articulation chaque sommet de degré $\mathrm{n}$ appartient à $\mathrm{n}$ faces finies ou non.

Nous dirons qu'un sommet de degré $\mathrm{n}$ de $\mathrm{K}$ est intérieur s'il appartient à $\mathrm{n}$ faces finies. Le terme sidi sera utilisé pour désigner un sommet intérieur de degré impair. Une arête de K sera dite intérieure si elle délimite deux faces finies. Dans le cas 
contraire elle sera dite extérieure. Une arête extérieure délimite une face finie et la face infinie.

Un sommet intérieur sera dit de profondeur 1 s'il est adjacent à au moins un sommet extérieur. Un sommet intérieur sera dit de profondeur $n(n>1)$ s'il est adjacent à au moins un sommet de profondeur n-1 et n'est adjacent à aucun sommet de profondeur inférieur à $\mathrm{n}-1$. Pour un sommet intérieur $\mathrm{S}$ de profondeur $\mathrm{n}$ il existe donc au moins une chaîne de $\mathrm{n}$ arêtes reliant $\mathrm{S}$ à un sommet extérieur.

\subsection{Circuit graphe et degré de retournement}

Un circuit graphe est une carte dont les arcs sont orientés de telle sorte que chaque face finie soit un circuit. Un même arc peut être orienté dans les deux sens. Nous appellerons un tel arc aods (arc orienté dans les deux sens). Nous utiliserons le terme arc pour désigner une arête orientée dans un seul sens.

Nous noterons $\mathrm{O}(\mathrm{K})$ le circuit graphe obtenu en effectuant une orientation $\mathrm{O}$ sur les arêtes de la carte $\mathrm{K}$ de telle sorte que chaque face finie soit un circuit. Dans ce circuit graphe nous aurons des arcs et éventuellement des aods.

Nous noterons $\mathfrak{I}(\mathrm{O}(\mathrm{K}))$ le nombre d'aods du circuit graphe $\mathrm{O}(\mathrm{K})$. Le degré de retournement $\delta(\mathrm{K})$ d'une carte $\mathrm{K}$ est défini de la façon suivante :

$$
\delta(\mathrm{K})=\min \left(\mathfrak{I}\left(\mathrm{O}_{\mathrm{i}}(\mathrm{K})\right)\right)=\mathfrak{I}\left(\mathrm{O}^{\sim}(\mathrm{K})\right)
$$

Dans cette définition $\mathrm{O}_{\mathrm{i}}$ désigne une orientation possible des arêtes de $\mathrm{K}$ de telle sorte que chaque face de $\mathrm{K}$ soit un circuit. $\mathrm{O}^{-}$désigne une orientation des arêtes de $\mathrm{K}$ dans laquelle il y a le minimum d'aods. Un circuit graphe $\mathrm{O}^{-}(\mathrm{K})$ qui possède le nombre minimum d'aods pour la carte $\mathrm{K}$ sera appelé circuit graphe minimum de K. Nous avons montré que $\delta(\mathrm{K})$ est un entier positif ou nul borné comme indiqué ci-après [13]:

$$
\frac{n}{2} \leq \delta(K) \leq \sum_{i=1}^{n} p_{i}
$$

Dans cette relation $\mathrm{n}$ désigne le nombre de sidis de la carte $\mathrm{K}$ et $\mathrm{p}$ désigne la profondeur du sidi i.

Une chaîne constituée d'aods sera appelée caods (chaine d'arcs orientés dans les deux sens) si et seulement si elle relie un sidi à un sommet extérieur ou un sidi à un autre sidi et n'admet aucun sommet extérieur comme sommet intermédiaire.

Nous dirons qu'une face d'un circuit graphe est de sens 0 si l'observateur placé dans cette face voit les arcs de cette face orientés dans le sens des aiguilles d'une montre. Dans le cas contraire nous dirons que la face est de sens 1 . Nous supposons dans cette définition que chaque face d'un circuit graphe a au moins un arc dont le sens 
détermine le sens de la face. Nous montrerons plus loin dans cet article que cette proposition est vraie pour les circuits graphe minimums.

\subsection{Remarque}

Le degré de retournement d'une carte est une fonction entre autre du nombre de sidi de cette carte. Une carte sans sidi est à zéro degré de retournement [11]. Ce résultat fait penser au problème de recherche d'un chemin eulérien dans un graphe et particulièrement au problème du postier chinois [2], [9] qui eux aussi font référence aux sommets de degré impair. Il y a cependant quelques différences:

- La recherche du degré de retournement concerne les graphes planaires topologiques avec la notion de face. Cette contrainte n'existe pas sur le problème du postier chinois qui peut se poser sur un graphe quelconque (avec isthme et ou point d'articulation par exemple).

- Le problème de recherche du degré de retournement impose une contrainte locale sur chaque face (être un circuit), alors que celui du postier chinois impose une contrainte sur l'ensemble des arcs du graphe (chaque arc doit être parcouru au moins une fois).

- Le problème du postier chinois suppose au départ un graphe orienté avec éventuellement des arcs orientés dans les deux sens (rue d'une ville que le postier doit emprunter). Le problème du degré de retournement part d'un graphe non orienté et recherche une orientation de ce graphe.

Toute carte qui est un graphe eulérien est à zéro degré de retournement mais le contraire n'est pas vrai comme le montre le fameux graphe des ponts de Koenigsberg [18] figure 1, qui est à zéro degré de retournement mais qui ne possède pas de chemin eulérien.

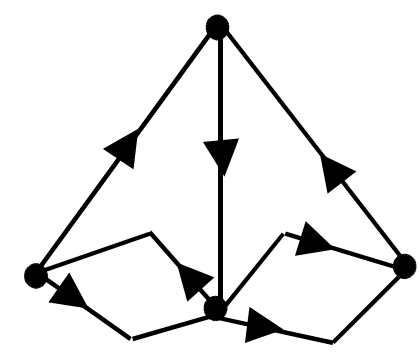

Figure 1. Exemple de circuit graphe minimum 
De même un chemin optimal du postier sur une carte ne fait pas de cette carte un circuit graphe minimum comme le montre la figure 2 où on suppose que tous les arcs ont le même poids.

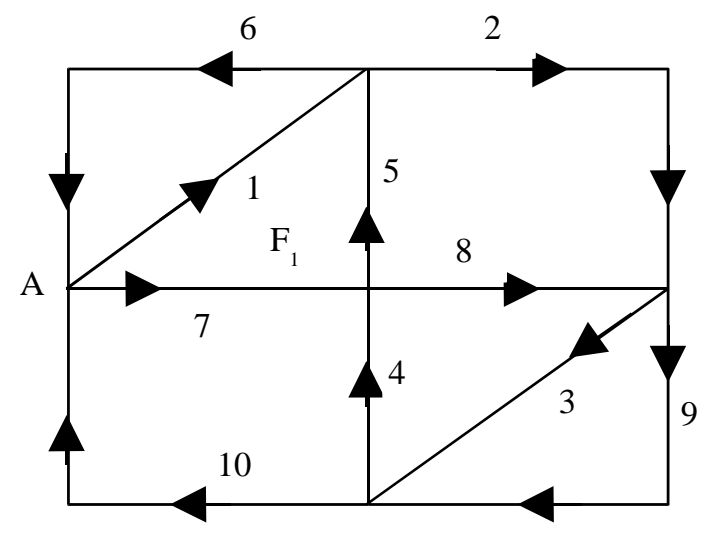

Figure 2. Exemple de graphe à cycle eulérien

Le cycle eulérien du graphe de la figure 2 est obtenu en partant de A par exemple et en suivant dans le sens indiqué, les arcs numérotés de 1 à 10 . Ce graphe n'est pas un circuit graphe car la face $F_{1}$ par exemple n'est pas un circuit.

La carte $\mathrm{K}$ de la figure 3 avec le sommet $\mathrm{E}$ origine de quatre arcs est un circuit graphe minimum. La carte est à zéro degré de retournement. Si nous considérons qu'il s'agit d'un graphe non planaire avec les arcs $\mathrm{AC}$ et $\mathrm{BD}$ qui n'ont aucun point commun (pas de contact au point E), les faces de $\mathrm{K}$ ne sont pas des circuits. La face $\mathrm{ABC}$ par exemple n'est pas un circuit même si on considère que l'arc AC est orienté dans les deux sens. Les résultats présentés dans cet article concernent uniquement les graphes planaires topologiques connexes sans isthmes et sans point d'articulation comme précisé plus haut.

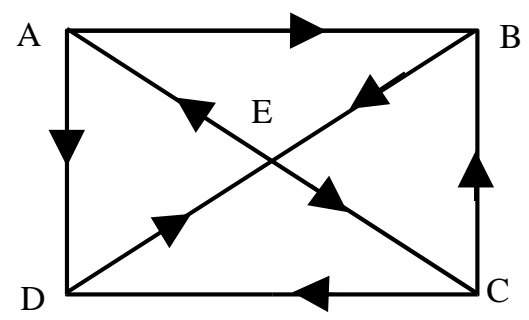

Figure 3. Graphe planaire et graphe non planaire

A R I M A 


\section{Propriétés d'un circuit graphe minimum}

Dans cette partie nous présentons quelques propriétés d'un circuit graphe minimum.

\subsection{Proposition 1}

Dans un circuit graphe deux faces voisines séparées par un arc sont de sens différents.

Cette proposition est une conséquence de la définition du sens d'une face telle que nous pouvons le voir dans la carte de la figure 4.

Figure 4. Exemple de circuit graphe minimum

La figure 4 montre un exemple de circuit graphe minimum [11], [13], [14] composé de trois faces $F_{1}, F_{2}$ et $F_{3}$. La face $F_{1}$ a le sens contraire des aiguilles d'une montre (sens 1). Les faces $F_{2}$ et $F_{3}$ ont le sens des aiguilles d'une montre (sens 0 ). Les faces $F_{1}$ et $F_{2}$ sont délimitées par un arc. Cette carte est à 1 degré de retournement.

Notons qu'une carte qui a deux faces finies est à zéro degré de retournement [11]. Ceci n'est pas vrai pour tout graphe planaire topologique comme le montre la figure 2.

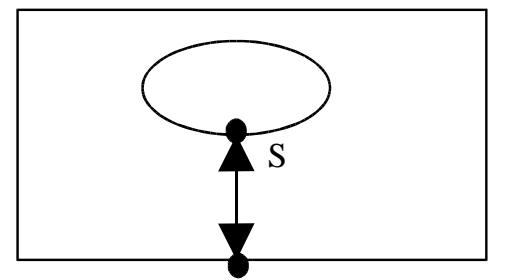

Figure 5. Exemple de graphe planaire à 2 faces et à 1 degré de retournement

La figure 5 montre un graphe planaire topologie contenant un isthme, une boucle et un sidi qui est en même temps un point d'articulation. Ce graphe qui a deux faces finies est à 1 degré de retournement. En effet la présence d'un sidi fait que le graphe ne peut être à 0 degré de retournement. 


\subsection{Proposition 2}

Dans un circuit graphe minimum deux faces voisines séparées par un aods ont le même sens.

Preuve : Supposons que dans un circuit graphe minimum $\mathrm{O}^{-}(\mathrm{K})$ deux faces voisines séparées par un aods an soient de sens différent. Remplaçons l'aods a par un arc en préservant le sens de chaque face. Ceci est possible à cause de la proposition 1 . Le circuit graphe $\mathrm{O}^{\prime}(\mathrm{K})$ obtenu avec la nouvelle orientation aura un aods en moins ce qui est contraire à l'hypothèse puisque le circuit graphe initial était minimum pour la carte K. Donc deux faces voisines séparées par un aods ont le même sens .

L'arc séparant les faces $\mathrm{F}_{2}$ et $\mathrm{F}_{3}$ de la figure 4 est orienté dans les deux sens et les deux faces ont le même sens qui est 0 .

\subsection{Proposition 3}

Dans un circuit graphe minimum aucune arête extérieure n'est un aods.

Preuve : Supposons qu'il existe un circuit graphe minimum $\mathrm{O}^{-}(\mathrm{K})$ qui possède une arête extérieure $\mathrm{a}_{0}$ orientée dans les deux sens. Cette arête délimite une face finie $\mathrm{F}$ et la face infinie. $\mathrm{O}^{\sim}(\mathrm{K})$ étant un circuit graphe minimum la face infinie n'est pas forcément un circuit. Nous pouvons dont orienter $\mathrm{a}_{0}$ suivant le sens de $\mathrm{F}$ et obtenir un circuit graphe $\mathrm{O}^{\prime}(\mathrm{K})$ qui a moins d'aods que $\mathrm{O}^{\sim}(\mathrm{K})$, ce qui est contraire à l'hypothèse .

Une conséquence de cette proposition est que dans un circuit graphe minimum aucun sommet extérieur de degré deux ne peut être l'origine d'un aods. Autrement dit, tout sommet extérieur origine d'un aods est de degré strictement supérieur à deux.

Dans la recherche d'un circuit graphe minimum il faut donc éliminer d'office les cas d'orientation contenant des aods entre une face finie et la face infinie.

\subsection{Théorème 1}

Dans un circuit graphe minimum toute face finie a au moins un arc dont le sens d'orientation par définition est celui de cette face.

Preuve : Supposons qu'il existe un circuit graphe minimum $\mathrm{O}^{-}(\mathrm{K})$ possédant une face finie $\mathrm{F}$ uniquement délimitée par des aods. D'après la proposition 2, ceci veut dire que toutes les faces voisines de F ont le même sens que F. Soit s ce sens (s vaut 0 ou 1). Orientons toutes les arêtes de F dans le sens 1-s. Cette orientation maintient le sens des faces voisines et permet d'avoir un circuit graphe $\mathrm{O}^{\prime}(\mathrm{K})$ qui a moins d'aods que le circuit graphe initial $\mathrm{O}^{-}(\mathrm{K})$, lequel par hypothèse est minimum. Ce n'est pas possible. Nous en déduisons que la face $\mathrm{F}$ a au moins un arc . 
Cet important théorème suggère que dans la recherche des circuits graphe minimums il faut d'office éliminer les cas d'orientation pouvant contenir des faces finies délimitées uniquement par des aods.

\subsection{Proposition 4}

Si dans un circuit graphe minimum $\mathrm{O}^{-}(\mathrm{K})$ on change le sens de tous les arcs on obtient encore un circuit graphe minimum que nous appellerons circuit graphe minimum dual du premier et qui sera noté $\mathrm{O}^{\sim} \mathrm{d}(\mathrm{K})$.

Preuve : En changeant le sens de tous les arcs, chaque face change tout simplement de sens (passant du sens 1 au sens 0 ou du sens 0 au sens 1). Le nombre d'aods reste inchangé puisque cette opération ne touche aucun aods. Le nouveau circuit graphe obtenu est donc aussi minimum .

Ce résultat montre que pour chaque carte il existe au moins deux circuits graphe minimums. Il peut en exister plusieurs. Dans [11] nous avons montré que le degré de retournement d'une carte qui n'a pas de sidi est nul. Pour une telle carte nous aurons uniquement deux circuits graphe minimums dual l'un de l'autre.

\subsection{Proposition 5}

Dans un circuit graphe minimum tout sidi est incident à $2 \mathrm{n}+1$ aods ; $\mathrm{n}$ étant un entier positif ou nul.

Preuve : Soit $\mathrm{S}$ un sidi d'un circuit graphe minimum $\mathrm{O}^{-}(\mathrm{K}) . \mathrm{S}$ est incident à $2 \mathrm{~m}+1$ arêtes $(\mathrm{m}>0)$. Ces arêtes délimitent $2 \mathrm{~m}+1$ faces finies que nous pouvons numéroter de $F_{1}$ à $F_{2 m+1}$ de telle sorte que $F_{1}$ soit voisine de $F_{2}, F_{2}$ voisine de $F_{3}, \ldots, F_{2 m+1}$ voisine de $F_{1}$. Supposons que $\mathrm{S}$ soit incident à $2 \mathrm{n}$ aods dans $\mathrm{O}^{\sim}(\mathrm{K})$, n étant positif ou nul.

Parcourons toutes les $2 \mathrm{~m}+1$ faces dans l'ordre de la numérotation en commençant par la première face et en terminant par cette même première face. Pour passer de la face $F_{t}$ à la face $F_{t+1} \quad(1 \leq t \leq 2 m+1)$ nous ferons un changement de sens si et seulement si les deux faces ont des sens différents; de même en passant de la face $\mathrm{F}_{2 \mathrm{~m}+1}$ à la face $F_{1}$. Au départ le sens est celui de $F_{1}$. Comme $S$ a $2 m+1$ arêtes nous ferons $(2 m+1)-2 n$ changements de sens soit $2(m-n)+1$ changements. Ce qui fait un nombre impair de changements. Ceci n'est possible que si $\mathrm{F}_{1}$ a deux sens d'orientation. Or par définitions chaque face n'a qu'un seul sens (0 ou 1). Nous en déduisons que $S$ ne peut pas être incident à un nombre pair d'aods. Il est donc incident à un nombre impair d'aods.

La même approche permet de montrer que le nombre d'aods est au moins égal à 1 . 


\subsection{Proposition 6}

Dans un circuit graphe minimum tout sommet intérieur $\mathrm{S}$ de degré pair est l'origine de $2 \mathrm{n}$ aods, $\mathrm{n}$ étant un entier positif ou nul.

La démonstration de cette proposition est similaire à celle de la proposition 5 .

Nous pouvons résumer les propositions 5 et 6 en disant que dans un circuit graphe le parcours des différentes faces auxquelles appartient un sommet $S$ (de $F_{1}$ à $F_{1}$ ) nécessite un nombre pair de changements d'orientation. Pour que ce nombre pair d'orientations soit respecté autour d'un sidi il faut au moins un aods dont la traversée n'implique pas de changement d'orientation. Cette aods élimine pour ainsi dire l'effet du sidi ou l'effet d'imparité introduit par le sidi. Si cet aods qui part du sidi se termine sur un sommet intérieur de degré pair, il introduit une imparité sur ce sommet. Pour éliminer cette imparité il faut qu'un second aods parte de ce sommet intérieur de degré pair. Ce processus s'achève sur un sidi ou sur un sommet extérieur.

\subsection{Théorème 2}

Si dans une carte $\mathrm{K}$ chaque sidi est incident à un nombre impair d'aods et chaque sommet intérieur de degré pair incident à un nombre pair (ou nul) d'aods, alors il existe une orientation $\mathrm{O}$ qui n'introduit pas de nouvel aods et telle que $\mathrm{O}(\mathrm{K})$ soit un circuit graphe.

Preuve : Soit F une face quelconque de K. Imposons à cette face un sens ( 0 ou 1$)$. Prenons ensuite un sommet de $\mathrm{F}$ et parcourons dans un sens donné (celui des aiguilles d'une montre par exemple) les différentes faces auxquelles appartient le sommet S. Le sens de la face $\mathrm{F}$ va imposer un sens à chacune de ces faces. Ceci est une conséquence des propositions 5 et 6 . En reprenant la même opération pour les autres sommets des différentes faces déjà orientées, on oriente de proche en proche toutes les autres faces finies de la carte. Notons que cette orientation n'introduit aucun nouveau aods. Le circuit graphe obtenu n'est pas forcément minimum.

Un cas particulier d'application de ce théorème est le cas des cartes qui n'ont pas de sidi. Les différents sommets intérieurs sont de degré pair. Ces cartes sont à zéro degré de retournement [11]. Ce qui veut dire qu'il existe au moins une orientation qui n'a pas d'aods et qui fait d'une telle carte un circuit graphe minimum.

\subsection{Proposition 7}

Dans un circuit graphe minimum une caods ne passe pas deux fois par un même sommet.

Preuve : Supposons qu'il existe un circuit graphe minimum $\mathrm{O}^{-}(\mathrm{K})$ dans lequel une caods $\mathrm{C}_{0}$ passe deux fois par un sommet $\mathrm{S}_{0}$ comme le montre la figure 6 . 


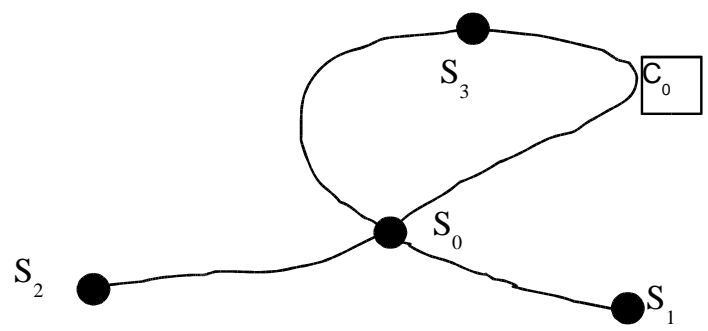

Figure 6. Chaîne d'aods passant deux fois par un même sommet So

Considérons la carte $\mathrm{K}^{\prime}$ avec les mêmes aods que $\mathrm{O}^{-}(\mathrm{K})$ sauf les aods de la boucle passant par les sommets $S_{0}, S_{3}$ et $S_{0}$ qui sont de simples arêtes non orientées de K'. Par construction cette carte vérifie les conditions du théorème 2 . Il existe par conséquent une orientation de $K^{\prime}$ telle que $\mathrm{O}^{\prime}\left(\mathrm{K}^{\prime}\right)$ soit un circuit graphe. Par construction $\mathrm{K}^{\prime}$ n'est pas différent de la carte $\mathrm{K}$. L'orientation $\mathrm{O}^{\prime}$ de $\mathrm{K}^{\prime}$ est donc une orientation de $\mathrm{K}$. O'(K) a moins d'aods que $\mathrm{O}^{\sim}(\mathrm{K})$, ce qui est contraire à l'hypothèse. Une caods n'a donc pas de boucle .

Nous en déduisons que dans un circuit graphe minimum une caods ne peut partir d'un sommet et revenir se terminer sur le même sommet, car en supprimant cette boucle on pourrait construire un circuit graphe avec moins d'aods.

De même dans un circuit graphe minimum deux caods distinctes ne peuvent se rencontrer en plus d'un point.

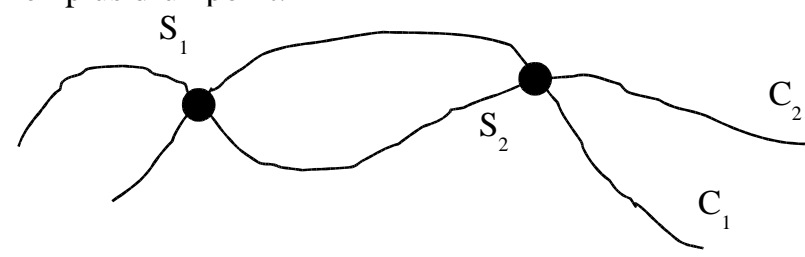

Figure 7. Chaînes d'aods se rencontrant en deux points

En effet si les chaînes $C_{1}$ et $C_{2}$ de la figure 7 étaient des caods dans un circuit graphe minimum, on pourrait enlever les aods de la boucle passant par $S_{1}$ et $S_{2}$ et avoir un circuit graphe avec moins d'aods. Ce qui serait contraire à l'hypothèse.

\subsection{Théorème 3}

Dans un circuit graphe minimum tout aods appartient à au moins une caods.

Preuve : Pour démontrer ce théorème nous allons énumérer les différents cas possibles. Un aods peut relier deux sidis, un sidi à un sommet extérieur, deux sommets 
extérieurs, un sommet intérieur de degré pair à un sidi, un sommet intérieur de degré pair à un sommet extérieur ou deux sommets intérieurs de degré pair entre eux :

- Si l'aods considéré relie deux sidis elle appartient à la caods constituée de cette arête unique.

- Il en est de même si l'aods relie un sidi à un sommet extérieur.

- D'après la proposition 3 un aods ne peut pas relier deux sommets extérieurs dans un circuit graphe minimum.

- Si un aods $\mathrm{a}_{0}$ relie un sidi à un sommet intérieur de degré pair $\mathrm{S}_{1}$, ce sommet $\mathrm{S}_{1}$ a au moins un autre aods $a_{1}$ qui lui est incident à cause de la proposition 6 . Si l'autre extrémité de $\mathrm{a}_{1}$ est un sidi ou un sommet extérieur, $\mathrm{a}_{0}$ appartient à la caods formée par $a_{0}$ et $a_{1}$. Si l'autre extrémité de $a_{1}$ est aussi un sommet $S_{2}$ de degré pair on reprend le processus jusqu'à trouver un sidi ou un sommet extérieur. Le nombre de sommets étant fini et une caods n'ayant pas de circuit on finira par tomber sur un sidi ou sur un sommet extérieur. L'arête $\mathrm{a}_{0}$ appartiendra à la caods constituée par la chaîne d'aods ainsi obtenue.

- Le cas où un aods relie un sommet extérieur à un sommet intérieur de degré pair est tout à fait similaire au cas précédent, le sommet extérieur remplaçant ici le sidi du cas précédent.

- Si un aods relie deux sommets intérieurs de degré pair $S_{1}$ et $S_{2}$, chacun de ces deux sommets est au moins l'origine d'un autre aods différent de a qui conduit soit à un sidi, soit à un sommet extérieur, soit enfin à un autre sommet intérieur de degré pair. Comme nous l'avons vu plus haut, la chaîne d'aods progressivement constituée de cette façon est une caods .

\section{Quelques remarques}

Les différentes propriétés ci-dessus suggèrent un certain nombre de remarques que nous allons présenter dans cette partie. Ces remarques portent sur la disposition des sidis les uns par rapport aux autres. Comme la disposition des sidis intervient dans le calcul du degré de retournement, ces remarques peuvent, elles aussi, suggérer à la longue certaines heuristiques.

\subsection{Remarque 1}

Un même sommet extérieur peut être l'extrémité de plusieurs aods comme le montre la figure 8 .

\section{A R I M A}




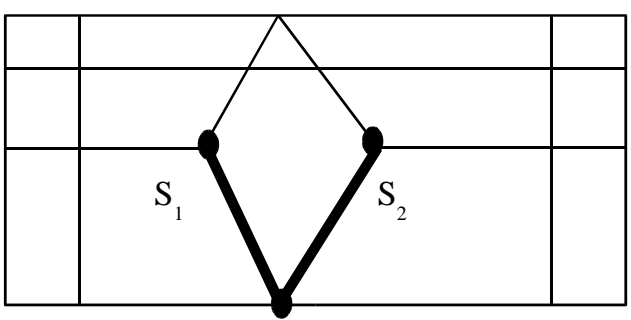

Figure 8. Deux aods issus d'un $\mathrm{S}_{0}$ me sommet extérieur

Dans la figure 8 l'effet d'imparité introduit par chacun des sidis $S_{1}$ et $S_{2}$ est éliminé en reliant ce sidi au sommet extérieur $\mathrm{S}_{0}$. Nous dirons aussi que l'effet d'imparité de chacun des sidis $S_{1}$ et $S_{2}$ est éliminé en les isolant. Ainsi les arcs $\left(S_{1}, S_{0}\right)$ et $\left(S_{0}, S_{2}\right)$ sont orientés dans les deux sens, ce sont des aods. Dans le cas où un sidi est relié à un autre sidi par une caods nous dirons que les deux sidis sont appariés. Eliminer l'effet d'imparité d'un sidi sera effectué soit en l'isolant, soit en l'appariant à un autre sidi.

\subsection{Remarque 2}

Un sidi peut être l'origine de plusieurs aods comme le montre la figure 9. Cette figure montre un exemple de carte qui a quatre sidis $S_{1}, S_{2}, S_{3}$ et $S_{4}$. En orientant les arêtes $\left(S_{1}, S_{2}\right),\left(S_{2}, S_{3}\right)$ et $\left(S_{2}, S_{4}\right)$ dans les deux sens on a un circuit graphe minimum $\mathrm{O}^{-}$ (K). Le degré de retournement de cette carte est de 3 [13]. 


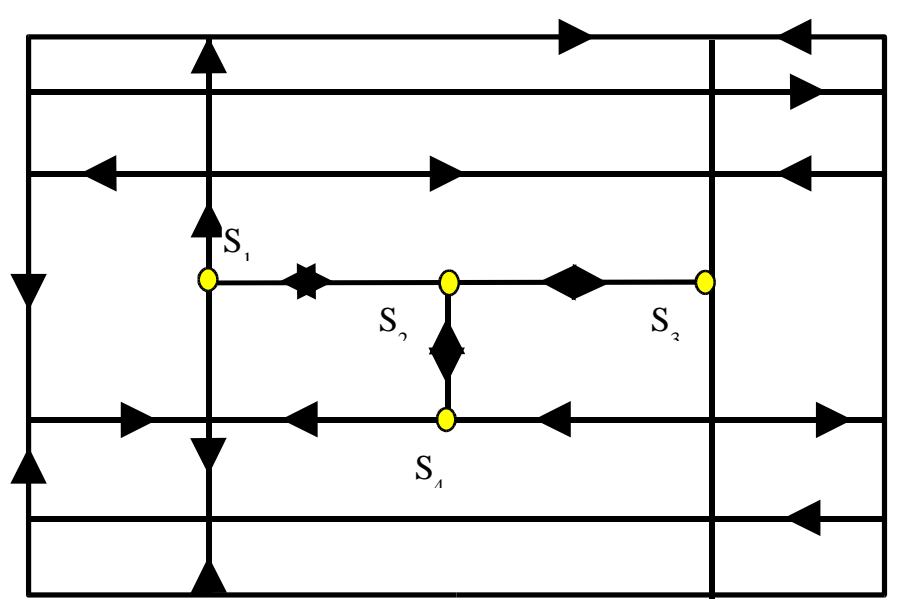

Figure 9. Carte à 4 sidis et à 3 degrés de retournement

\subsection{Remarque 3}

Considérons le schéma de la figure 10. Nous supposons que les arêtes de ce graphe représentent les aods d'un circuit graphe minimum $\mathrm{O}^{-}(\mathrm{K})$ non entièrement représenté. Nous supposons aussi que $S_{11}, S_{12}, S_{21}$ et $S_{22}$ ne sont l'origine que d'un seul aods chacun. Le sommet $S_{11}$ par exemple est l'origine du seul aods $\left(S_{11}, S\right)$. Nous allons montrer que les quatre sommets $S_{11}, S_{12}, S_{21}$, et $S_{22}$ sont tous des sidis.

Les sommets $S_{11}, S_{12}, S_{21}$ et $S_{22}$ étant l'origine d'un seul aods chacun, ne sont pas des sommets intérieurs de degré pair d'après la proposition 6.

Les sommets $S_{11}$ et $S_{12}$ ne peuvent pas être tous les deux des sommets extérieurs. Il en est de même des sommets $S_{21}$ et $S_{22}$. En effet si $S_{11}$ et $S_{12}$ sont tous les deux des sommets extérieurs on peut construire (théorème 2 ) un circuit graphe $\mathrm{O}^{\prime}(\mathrm{K})$ dans lequel les arêtes $\left(\mathrm{S}, \mathrm{S}_{11}\right)$ et $\left(\mathrm{S}, \mathrm{S}_{12}\right)$ sont orientées dans un seul sens. Ce graphe aurait moins d'aods que $^{-}(\mathrm{K})$ ce qui est impossible. Les sommets $S$ et $S^{\prime}$ peuvent être des sidis ou des sommets intérieurs de degré pair. S'ils sont des sommets intérieurs de degré pair cela veut dire que chacun d'eux a un autre aods qui n'est pas représenté sur le schéma d'après la proposition 6 .

Supposons que $S_{11}$ soit un sidi et $S_{12}$ un sommet extérieur et de même de l'autre côté $S_{21}$ soit un sidi et $S_{22}$ un sommet extérieur. Considérons cette fois la carte K' qui a les mêmes aods que $\mathrm{O}^{\sim}(\mathrm{K})$ sauf les aods $\left(\mathrm{S}_{12}, \mathrm{~S}^{\prime}\right),\left(\mathrm{S}^{\prime}, \mathrm{S}^{\prime}\right)$ et $\left(\mathrm{S}^{\prime}, \mathrm{S}_{22}\right)$ qui sont de simples arêtes dans $\mathrm{K}^{\prime}$. Comme nous avons enlevé deux aods à chaque sommet, $\mathrm{K}^{\prime}$ vérifie encore les conditions du théorème 2. Il existe une orientation $\mathrm{O}^{\prime}$ telle que $\mathrm{O}^{\prime}(\mathrm{K})$ soit un 
circuit graphe avec moins d'aods que $\mathrm{O}^{-}(\mathrm{K})$, ce qui n'est pas possible d'après l'hypothèse.

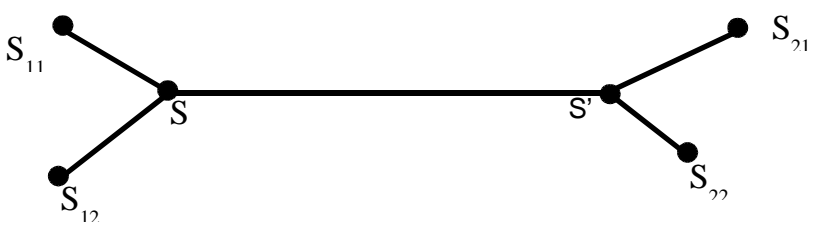

Figure 10. Exemple de disposition d'aods dans un circuit graphe minimum

Nous avons montré que $S_{11}$ et $S_{12}$ ne sont pas des sommets intérieurs de degré pair. Ils ne sont pas tous les deux des sommets extérieurs (il y a au moins un sidi de chaque côté), l'un ne peut être un sidi et l'autre un sommet extérieur. Les deux sommets sont donc des sidis. Il en est de même de $S_{21}$ et de $S_{22}$.

On peut interpréter de plusieurs façons ce schéma:

- Tous les 6 sommets représentés sont des sidis appariés deux à deux. $S_{11}$ est apparié à $\mathrm{S}_{12}, \mathrm{~S}$ est apparié à $\mathrm{S}$ ' et $\mathrm{S}_{21}$ est apparié à $\mathrm{S}_{22}$. Dans cette interprétation nous avons 3 caods qui, deux à deux, n'ont aucun aods en commun.

- $\mathrm{S}$ et $\mathrm{S}$ ' sont des sommets intérieurs de degré pair. Il existe alors pour chacun de ces deux sommets un autre aods différent de $\left(\mathrm{S}, \mathrm{S}^{\prime}\right)$ qui lui est incident et qui n'est pas représenté. Les sidis $S_{11}$ et $S_{12}$ sont appariés ainsi que $S_{21}$ et $S_{22}$.

- $S$ est un sommet extérieur, $S$ ' est un sidi comme les quatre autres. Dans ce cas les sidis $S_{11}, S_{12}$ et $S^{\prime}$ sont isolés. Les sidis $S_{21}$ et $S_{22}$ sont appariés.

Les deux sommets $S$ et $S$ ' ne peuvent tous les deux être des sommets extérieurs car deux sommets extérieurs ne peuvent être reliés par un aods dans un circuit graphe minimum (Proposition 3).

Dans ces différentes façons d'interpréter, deux caods n'ont pas d'aods commun et un sidi est l'origine d'une et d'une seule caods. D'autres caods peuvent cependant traverser le sidi.

La figure 11 montre un exemple de sommet intérieur $\mathrm{S}$ de degré pair qui est traversé par deux caods. Une caods horizontale et une caods verticale. Les deux caods ont uniquement un sommet commun. Chacune des deux caods est constituée de deux aods. Notons qu'il existe d'autres façons d'interpréter avec toujours deux caods. La carte correspondante est de degré de retournement 4. 


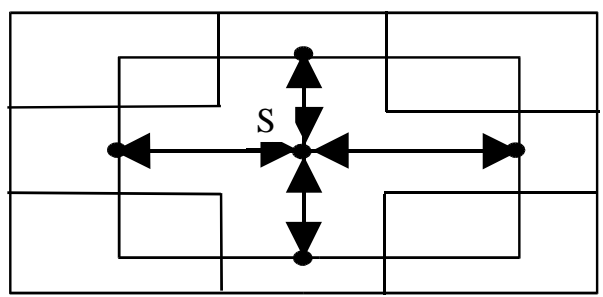

Figure 11. Exemple de sommet de degré pair d'où partent 4 aods

\subsection{Remarque 4}

Le nombre de circuits graphe minimums associés à une carte $\mathrm{K}$ (nous le notons $\phi$ (K)) peut être très élevé même quand cette carte a peu de sidi. La carte de la figure 12 admet dix circuits graphe minimums correspondant aux cinq possibilités d'isolement de son unique sidi.

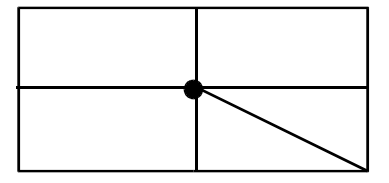

Figure 12. Carte à un sidi et à 10 circuits graphe minimums

Le nombre de sidi d'une carte n'est cependant pas en liaison direct avec le nombre de circuits graphe minimums associés à cette carte.

\section{Notions de parcours et d'interprétation}

Les différentes remarques faites dans la section précédente font ressortir deux notions, la recherche d'une caods à laquelle appartient un aods et l'interprétation de la disposition des caods dans un circuit graphe minimum. Dans cette partie nous précisons la notion de parcours d'aods dans un circuit graphe minium et nous montrons que chaque parcours d'aods génère une caods. Nous introduisons aussi la notion d'interprétation d'un circuit graphe minimum et nous montrons que pour tout circuit graphe minimum il existe au moins une interprétation cohérente. Il peut en exister plusieurs comme évoqué plus haut dans les remarques. 


\subsection{Notion de parcours dans un circuit graphe minimum}

Un parcours d'aods commence par un aods quelconque ao. On passe ensuite à un aods adjacent au premier, puis à un aods adjacent au second et ainsi de suite. Le parcours a deux parties, le parcours arrière et le parcours avant. Le parcours arrière est celui qui est fait à partir de l'origine (choisie de façon arbitraire) de l'aods a et le parcours avant est fait à partir de l'autre extrémité de ao.

Un parcours est normal si on s'arrête dès qu'on rencontre un sidi ou un sommet extérieur, ceci que ce soit dans la partie parcours arrière ou dans la partie parcours avant. Si dès le départ l'origine de an est un sidi ou un sommet extérieur, le parcours arrière s'arête à ce sommet. Il en est de même de l'autre extrémité de a pour le parcours avant.

Le résultat des deux parcours arrière et avant est une chaine d'aods contenant a que nous noterons $\mathrm{Ca}_{0}$ (chaîne d'aods résultante du parcours de l'aods $\mathrm{a}_{0}$ ).

\subsection{Théorème 4}

Dans un circuit graphe minimum la chaîne d'aods résultante de tout parcours normal d'aods est une caods.

Preuve : Par construction la chaîne $\mathrm{Ca}_{0}$ d'aods (de la carte $\mathrm{K}$ ) obtenue se termine soit par deux sidis (c'est une caods par définition), soit par un sidi et un sommet extérieur (c'est encore une caods), soit enfin par deux sommets extérieurs. Montrons que ce dernier cas n'est pas possible.

En effet si on considère la carte K' qui a les mêmes aods que $\mathrm{K}$ sauf les aods de la chaîne $\mathrm{Ca}_{0}, \mathrm{~K}^{\prime}$ vérifie les conditions du théorème 2 , ce qui impliquerai que $\mathrm{O}(\mathrm{K})$ n'est pas un circuit graphe minimum sur $\mathrm{K}$, résultat contraire à l'hypothèse. Les deux extrémités ne peuvent donc être des sommets extérieurs. La chaîne Cas obtenue est une caods .

Notons que pour un aods $\mathrm{a}_{0}$ donné, $\mathrm{Ca}_{0}$ n'est pas forcément unique. Il peut y en avoir plusieurs. D'après ce théorème tout se passe comme si dans un circuit graphe minimum il n'y a que des caods.

\subsection{Notion d'interprétation d'un circuit graphe minimum}

Soit $\mathrm{O}^{\sim}(\mathrm{K})$ un circuit graphe minimum possédant au moins un sidi. Soit $\mathrm{Y}$ $l^{\prime}$ 'ensemble des sidis de $\mathrm{O}^{-}(\mathrm{K})$. Une interprétation cohérente sur $\mathrm{O}^{-}(\mathrm{K})$ est une partition de $\mathrm{Y}$ en deux sous-ensembles $\mathrm{Y}_{1}$ et $\mathrm{Y}_{2}$ tels que :

$\mathrm{Y}=\mathrm{Y}_{1} \cup \mathrm{Y}_{2}$

Si $\mathrm{s} \in \mathrm{Y}_{1}$ alors $\mathrm{s} \notin \mathrm{Y}_{2}$ 
Si $\mathrm{s} \in \mathrm{Y}_{1}$ alors $\mathrm{s}$ est un sidi isolé

Si $\mathrm{s} \in \mathrm{Y}_{2}$ alors il existe s' $\in \mathrm{Y}_{2}$ tel que s soit apparié à s'.

Pour un circuit graphe minimum donné il peut y avoir plusieurs interprétations cohérentes. Nous dirons que toutes les interprétations cohérentes sur un même circuit graphe minimum sont équivalentes. Dans la recherche du degré de retournement il suffira d'examiner une seule des interprétations équivalentes.

Le circuit graphe minimum de la figure 9 a plusieurs interprétations cohérentes. L'une d'elles consiste à dire que l'effet du sidi $\mathrm{S}_{1}$ a été éliminé en l'appariant au sidi $\mathrm{S}_{2}$ et l'effet du sidi $S_{3}$ a été éliminé en l'appariant au sidi $S_{4}$. On peut aussi dire que le sidi $S_{1}$ a été apparié au sidi $S_{4}$ et le sidi $S_{2}$ au sidi $S_{3}$. On peut dire enfin que le sidi $S_{1}$ a été apparié au sidi $S_{3}$ et le sidi $S_{2}$ au sidi $S_{4}$. Dans ces différentes interprétations $Y_{1}$ est vide.

\subsection{Théorème 5}

Etant donné un circuit graphe minimum $\mathrm{O}^{-}(\mathrm{K})$ possédant au moins un sidi il existe au moins une interprétation cohérente de ce circuit graphe minimum telle que deux caods n'aient aucun aods en commun.

Preuve : Soit un sidi $\mathrm{S}_{0}$ quelconque de $\mathrm{O}^{\sim}(\mathrm{K})$. $\mathrm{S}_{0}$ est l'origine d'au moins un aods $\mathrm{a}_{0}$ (Proposition 5). Le parcours de $\mathrm{a}_{0}$ donne une caods $\mathrm{Ca}_{0}$ qui permet soit d'isoler $\mathrm{S}_{0} \mathrm{si}$ l'autre extrémité est un sommet extérieur, soit de l'apparier à un autre sidi, ceci si l'autre extrémité est un sidi. Maintenons cette interprétation pour le sidi $\mathrm{S}_{0}$ et éventuellement la même interprétation pour le sidi $S_{0}{ }_{0}$ auquel il est apparié ( $\mathrm{S}_{0}$ appartient à $\mathrm{Y}_{1}$, ou $\mathrm{S}_{0}$ et $\mathrm{S}^{\prime}{ }_{0}$ appartiennent à $\mathrm{Y}_{2}$ ). Marquons tous les aods de $\mathrm{Ca}_{0}$ pour ne plus y revenir par la suite.

Si $\mathrm{S}_{0}$ est l'origine d'un seul aods, la visite de ce sommet est terminée. Si le sidi $\mathrm{S}_{0}$ est l'origine d'autres aods, il y en a en nombre pair. Nous allons les regrouper par deux : $a_{1}$ et $a_{1}{ }_{1}, a_{2}$ et a' ${ }_{2}$ et ainsi de suite. Le parcours normal de $a_{1}$ qui passe par a' ${ }_{1}$ est une caods (théorème 4). Elle permet soit d'isoler un autre sidi différents de $S_{0}$ et de $S^{\prime}{ }_{0}$ soit d'apparier deux autres sidis différents de $S_{0}$ et de $S^{\prime}{ }_{0}$. Nous retenons cette nouvelle interprétation pour le sidi isolé ou les deux sidis appariés et nous marquons les aods de la caods correspondante. Nous reprenons le processus pour les différents couples d'aods de $\mathrm{S}_{0}$ afin de finaliser la visite de ce sidi.

La même opération faite sur le sidi $\mathrm{S}_{0}$ est ensuite faite sur les sidis qui n'ont pas encore d'interprétation. En effet un sidi S qui n'a pas encore d'interprétation n'est sur aucune caods passant par les sidis déjà visités comme $\mathrm{S}_{0}$. $\mathrm{S}$ ne peut donc être apparié à un sidi déjà visité. $S_{0}$ ne peut être apparié à un autre sommet par la suite, car tout aods non marqué n'accepte plus ce sidi comme extrémité une fois qu'il a été visité. Nous construisons ainsi et de proche en proche une interprétation cohérente de $\mathrm{O}(\mathrm{K})$. Dans 
l'interprétation ainsi construite aucun aods n'appartient à deux caods car nous n'utilisons pas les aods déjà marqués pour construire de nouvelles caods. Appelons l'interprétation ainsi construite interprétation de base .

Pour un circuit graphe minimum il peut exister plusieurs interprétations de base suivant le sidi initial et l'aods initial de ce sidi. Dans la recherche du degré de retournement il serait intéressant d'examiner non pas les interprétations de base (différents cas d'appariements des sidis) mais le circuit graphe associé. Ce qui contribue à diminuer le nombre de cas à étudier.

\subsection{Corollaire 1}

Etant donné un circuit graphe minimum $\mathrm{O}^{-}(\mathrm{K})$ possédant au moins un sidi, il existe au moins une interprétation cohérente de $\mathrm{O}^{\sim}(\mathrm{K})$.

Preuve : Il suffit de construire une interprétation de base à partir d'un sidi de $\mathrm{O}(\mathrm{K})$

Ce résultat montre que dans la recherche du degré de retournement on peut se contenter d'examiner tous les cas d'appariement et d'isolement des différents sidis et en déduire celui qui a le minimum d'aods. Ce nombre minimum d'aods est le degré de retournement cherché.

\subsection{Théorème 6}

Etant donnés deux sidis $S$ et $S^{\prime}$ d'un circuit graphe minimum $\mathrm{O}^{-}(\mathrm{K})$ muni d'une interprétation de base $\mathrm{i}$, une seule des trois propositions suivantes est vraie :

$$
\begin{aligned}
& C_{S}^{i}=C_{S^{\prime}}^{i} \\
& C_{S}^{i} \cap C_{S^{\prime}}^{i}=\phi \\
& C_{S}^{i} \cap C_{S^{\prime}}^{i}=S^{\prime \prime}
\end{aligned}
$$

$\mathrm{C}_{\mathrm{s}}{ }^{\mathrm{i}}$ désigne la caods qui permet d'éliminer l'effet d'imparité du sidi $\mathrm{S}$ dans l'interprétation de base $\mathrm{i}$.

Preuve : C'est une conséquence directe du théorème 5. Dans la relation (1) S et S' sont des sidis appariés. Dans la relation (2) les deux sidis peuvent être soient isolés soient appariés chacun à un sidi différent. Dans la relation (3) S" désigne un sommet de $\mathrm{K}$.

S' est un sommet extérieur si $\mathrm{S}$ et $\mathrm{S}$ ' sont tous les deux isolés. S" est un sommet intérieur si au moins un des deux sidi $\mathrm{S}$ et $\mathrm{S}$ ' est apparié à un troisième sidi de $\mathrm{K}$.

En effet si $\mathrm{S}$ et S' sont isolés et ont un point commun, ce point ne peut être qu'un sommet extérieur sinon le circuit graphe ne serait pas minimum. Comme le montre la figure 13 . 


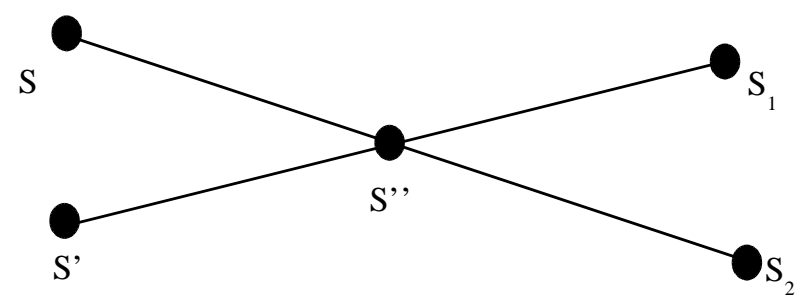

Figure 13. Deux sidis isolés par deux caods ayant un point commun $S^{\prime \prime}$

Sur la figure 13 nous supposons que $S$ et $S^{\prime}$ sont isolés et leurs deux caods ont un point commun $S$ ', qui n'est pas un sommet extérieur. $S_{1}$ et $S_{2}$ sont des sommets extérieurs qui à la limite peuvent être confondus. Selon le théorème 2 on peut construire un circuit graphe ne contenant pas les aods $\left(S{ }^{\prime}, S_{1}\right)$ et $\left(S^{\prime}{ }^{\prime}, S_{2}\right)$. Ce qui est contraire à l'hypothèse, car le circuit graphe initial est minimum. Les deux caods n'ont donc aucun sommet intérieur en commun.

De même si au moins un des deux sidis est apparié sa caods par définition ne passe par aucun sommet extérieur .

Signalons qu'une interprétation peut être cohérente sans être de base. Dans une interprétation non de base plusieurs caods peuvent avoir des aods en commun. Considérons en effet la figure 14.

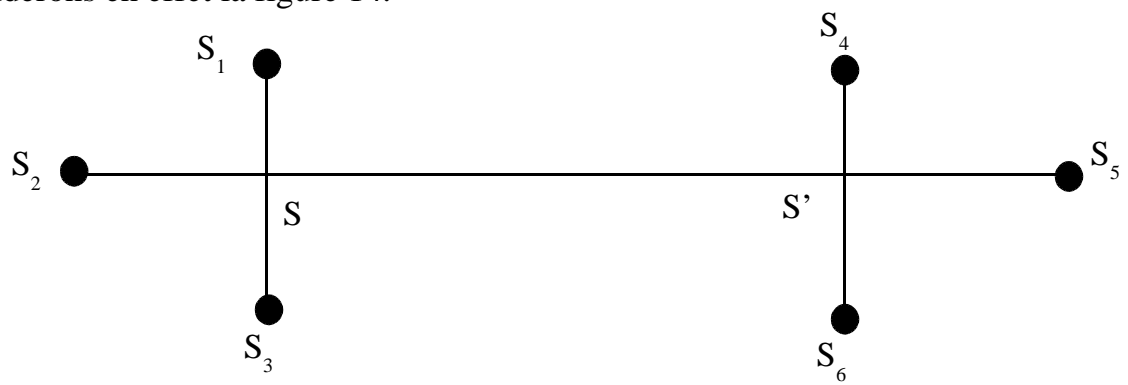

Figure 14. Carte à 6 sidis

Nous supposons qu'il s'agit sur cette figure 14 d'une carte dont seules les six sidis $S_{1}, S_{2}, S_{3}, S_{4}, S_{5}$ et $S_{6}$ sont représentés ainsi que deux sommets intérieurs de degré pair $S$ et $S^{\prime}$. Les traits représentent des aods. Une interprétation cohérente consiste à dire que les sidis sont appariés deux à deux : $S_{1}$ est apparié à $S_{4}$ par exemple, $S_{2}$ à $S_{5}$ et $S_{3}$ à $S_{6}$. Dans cette interprétation les trois caods qui en résultent ont un aods en commun qui est $\left(\mathrm{S}, \mathrm{S}^{\prime}\right)$.

Une interprétation de base partant de $S_{1}$ pourrait donner le résultat suivant : $S_{1}$ est apparié à $S_{2}, S_{3}$ est apparié à $S_{4}$ et $S_{5}$ est apparié à $S_{6}$. Dans cette interprétation les trois caods qui en résultent n'ont aucun aods en commun.

\section{A RI M A}


La recherche du degré de retournement d'une carte consiste à isoler ou apparier deux à deux les sidis de cette carte. Dans un circuit graphe minimum solution chaque sidi est donc soit isolé soit apparié à un autre. Ce résultat suggère que dans le cas de la figure 14 , si $S_{1}$ est déjà apparié à $S_{4}$, il ne faut plus apparier $S_{2}$ à $S_{5}$ car on va compter le même aods SS' deux fois. La solution obtenue ne sera pas optimale.

Comme il n'est généralement pas possible d'examiner directement les circuits graphe minimums dans la recherche du degré de retournement, il faudrait autant que possible examiner uniquement les interprétations de base en laissant de côté les autres interprétations qui ne conduisent pas à des solutions optimales bien que concernant parfois le même circuit graphe minimum.

\section{Conclusion}

Dans cet article nous avons défini la notion de circuit graphe et de circuit graphe minimum. Nous avons présenté quelques propriétés d'un circuit graphe minimum. Nous avons montré que dans un circuit graphe minimum aucune face n'est délimitée uniquement par des arcs orientés dans les deux sens. Ceci nous a permis de définir pour chaque face d'un circuit graphe minimum un sens d'orientation qui est celui d'au moins un arc délimitant cette face. Ce sens permet de mieux caractériser cette face et de la comparer à ses faces voisines. Nous avons aussi montré qui si dans une carte K chaque sidi (sommet intérieur de degré impair) est incident à un nombre impair d'aods (arc orienté dans les deux sens) et chaque sommet intérieur de degré pair incident à un nombre pair (ou nul) d'aods, alors il existe une orientation $\mathrm{O}$ qui n'introduit pas de nouvel aods et telle que $\mathrm{O}(\mathrm{K})$ soit un circuit graphe.

Nous avons étudié la disposition des arcs orientés dans les deux sens sur un circuit graphe minimum. Il en ressort entre autres qu'une chaîne constituée d'arcs orientés dans les deux sens n'a pas de circuit. Elle commence sur un sidi et finit soit sur un autre sidi soit sur un sommet extérieur. Elle peut traverser d'autres sommets intérieurs de degré pair ou impair. Nous avons enfin introduit la notion de parcours d'aods, d'interprétation cohérente et d'interprétation de base pour mieux appréhender les cas possibles pouvant être directement écartés lors de la recherche du degré de retournement.

Le degré de retournement d'un graphe planaire topologique est le nombre d'arcs orientés dans les deux sens d'un circuit graphe minimum associé à ce graphe. Trouver le degré de retournement d'un graphe c'est trouver un circuit graphe minimum associé à ce graphe. Une meilleure connaissance des propriétés des circuits graphe minimums permettra de mieux aborder le calcul de cette caractéristique des graphes, calcul connu pour son aspect explosion combinatoire. Ces connaissances ainsi que les remarques et suggestions faites tout au long de l'article aideront probablement à trouver des 
A RIM A - Volume 1-2005

algorithmes efficaces ou des heuristiques pouvant permettre de résoudre ce problème et d'en analyser la complexité.

\section{Bibliographie et biographie}

\subsection{Bibliographie}

[1] BERGE C., Graphes et hypergraphes, Dunod, 1970.

[2] EDMONDS J. Matching, Euler tours and the chinese postman, Mathematical Programming 5, 88-124, North-Holland Publishing Compagny, 1973.

[3] FRED S. ROBERTS, Graph Theory and its applications to problems of society, CBMS-NSF, Regional Conference Seties in Applied Mathematics, Vol 29, p. 7-13, Philadelphia, Pensylvania, 1978.

[4] GONDRAN M., MINOUX M., Graphes et Algorithmes, Eyrolles, 1979.

[5] INC (Institut National de Cartographie), Carte Administrative du Cameroun au 1/1500 000, édition, 1996.

[6] LAURINI R., MILLERET-RAFFORT F., Les bases de données en géomatique, Hermès, 1993.

[7] LAURINI R., THOMPSON D., Fundamentals of spatial information systems, The APIC series, Academic Press, 1995.

[8] LEVY G., Algorithme combinatoire, méthodes constructives, Dunod, 1994.

[9] LIN Y., ZHAO., A new algorithm for the directed chinese postman problem, Computers and Operations Research, 15(6), 577 - 584, 1988.

[10] NZALI J.P., TAPAMO H., Digitalisation au Millimètre, $3^{\circ}$ Colloque Africain sur la Recherche en Informatique (CARI'96), p. 237-246, Libreville, Gabon, Octobre 1996.

[11] NZALI J.P., TAPAMO H., Analyse de la Digitalisation au Millimètre, TSI, Vol $17, \mathrm{n}^{\circ}$ 6, Hermès, juin 1998 ,

[12] NZALI J.P., Degré de retournement d'une carte, Revue Internationale de Géomatique, Vol 9, n 2, Hermès Science 1999.

[13] NZALI J.P., KOUMPO TANEKOU P., TAPAMO H., Calcul du degré de retournement

A R I M A 
d'un graphe, $5^{\circ}$ Colloque Africain sur la Recherche en Informatique (CARI' 00), p. 57- 64, Antananarivo, Madagascar, Octobre 2000.

[14] NZALI J.P., KOUMPO TANEKOU P., TAPAMO H., Algorithme de calcul du degré de retournement d'un graphe planaire topologique, Revue Africaine de la Recherche en Informatique et Mathématiques Appliquées, ARIMA, Volume 1, 2002, pages 63 à 94 .

[15] OYSTEIN O., Graphs and their uses, Random House, The L.W. Singer Compagny, 1963.

[16] PORNON H., Utilisation et place des SIG dans les systèmes d'information des organisations, Revue de géomatique, Vol 3- $\mathrm{n}^{\circ}$ 1-2/1993, Hermès.

[17] SAKAROVITCH M., Optimisation combinatoire, programmation discrète, Hermann, Paris, 1984.

[18] SAKAROVITCH M., Optimisation combinatoire, graphe et programmation linéaire, Hermann, Paris, 1984.

\subsection{Biographie}

Nzali Jean Pierre est chargé de cours au département d'Informatique, Faculté des Sciences, Université de Yaoundé I, Cameroun. Depuis quelques années il s'intéresse au calcul du degré de retournement des graphes planaires topologiques. 